\title{
$U B V$ absolute CCD photometry and differential astrometry of close visual double stars, with G-type primaries ${ }^{\star, \star \star}$
}

\author{
T. Nakos ${ }^{1,2}$, D. Sinachopoulos ${ }^{1}$, and E. van Dessel ${ }^{1}$ \\ 1 Royal Belgian Observatory, Ring Laan 3, B-1180 Ukkel, Brussels, Belgium \\ 2 University of Thessaloniki, Department of Physics, Section of Astrophysics Astronomy and Mechanics, GR-54006 Thessaloniki, \\ Greece
}

Received May 29; accepted November 27, 1996

\begin{abstract}
We present $V$ magnitudes, $(B-V)$ and $(U-B)$ colours of close visual double star components, and their differences as well as separations and position angles of 40 visual double stars. The common properties of the members of this sample are the small angular separation $\rho$, which is less than $7^{\prime \prime}$, and the common spectral type of the primaries, which are of G-type. The observations made with the Bessel $U, B$ and $V$ filters, while the astrometry was performed in the $V$ filter only. For the observations, the CCD camera attached to the Cassegrain focus of the $90 \mathrm{~cm}$ Dutch telescope, at La Silla, Chile, had been used. From the analysis of the data we concluded that from the sample of the fourty double stars observed seven proved to be physical pairs, while the rest must have common origin components.
\end{abstract}

Key words: binaries: visual - catalog

\section{Introduction}

Until recently and due to technical difficulties (Rakos 1982) only few photometric measurements of close visual binaries have been published. For these reasons, our knowledge on this stellar system is still insufficient.

Under the visual binaries those systems with solar type components are especially interesting for understanding the evolution of the Sun. The present work is the first attempt to study them systematically: this is the first paper in which we present absolute photometry of close visual double stars with $\mathrm{G}$ - type primaries. The sample has been chosen in such a way that the secondaries are usually $\mathrm{G}$ - type stars as well.

Send offprint requests to: D. Sinachopoulos

* Based on observations made at ESO La Silla, Chile.

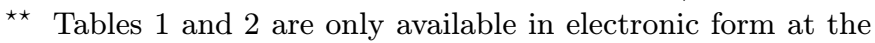
CDS via anonymous ftp to cdsarc.u-strasbg.fr (130.79.128.5) or via http://cdsweb.u-strasbg.fr/Abstract.html

\section{Observations}

The observations were carried out using the $90 \mathrm{~cm}$ Dutch telescope and its CCD camera at ESO La Silla, Chile, between the $25^{\text {th }}$ of April and $1^{\text {st }}$ of May, 1994. The ESO No. 29 CCD chip was installed in the camera. It is a chip of the type TEK $512 \mathrm{CB}$, thinned and AR coated for improved quantum efficiency. Its dimensions are $512 \times 512$ pixels of $27 \times 27$ microns each. Its linearity goes up to $265000 \mathrm{e}^{-} /$pixel remaining always better than $0.5 \%$, which makes it an excellent tool for observing relatively bright targets, as the binaries of our sample.

We have selected a fixed window $200 \times 200$ pixels wide on the CCD chip and we centered all double stars observed and the photometric standard stars used in this window before starting the exposures. We could save a lot of time reading out only this part of the CCD after each exposure. This improved significantly the efficiency of our observations. The window remained the same all nights. On the other hand, we used the whole surface of the CCD chip for the exposures aimed at its astrometric calibration.

According to the schedule followed, all visual binaries presented here were observed in only one filter during each night. Observing a target in different filters in the same night with CCDs is a time consuming method: as the focal length of the telescope depends on the filter properties, it is obvious that the instrument must be refocused whenever a different filter is used. On the contrary, using the same filter during the whole night increases significantly the efficiency of the observations.

Eight exposures per double star and the extinction star were taken in $V$ in the night of April 26 and four in the night of April 30. The duration of the exposures was as long as possible, at least two seconds and typically around five seconds. Eight exposures per double star and the extinction star were taken in $B$ in the night of April 27 and four in the night of May 1 . The duration of the exposures was at least three seconds and typically around six seconds. Finally, only three exposures per double star and 
the extinction star were taken in $U$ in the night of April 28 , since the duration of the exposures was between fifty and ninety seconds. The lower efficiency of the observations during the last three nights is due to the installation and tests of the new data acquisition system of the CCD camera, which is based on a new camera controller and a DEC Alpha workstation computer. We should note that at the 29th of April a lot of time was lost due to major problems of this newly installed data aquisition system of the telescope. In addition, the night was not photometric.

We preferred to use some of the stars in the central region of M 16 open cluster for the estimation of the scale of the CCD. For the calculation of the instrumental position angle of the CCD camera we used traces of stars in M 16 as well, taken on separate CCD frames.

During the first night (of April 25 to 26) 18 photometric standard stars from the $\mathrm{E}$ regions were observed, in all filters, for the calculation of the transformation coefficients into the $U B V$ system. Five exposures per filter were taken.

The atmospheric conditions under which the observations were carried out were very good. During all nights seeing at the $90 \mathrm{~cm}$ Dutch telescope was varying from 1.2 to 1.5 arcseconds but at the NTT (new technology telescope at ESO La Silla) was usually much better, while the temperature was fluctuating from $12{ }^{\circ} \mathrm{C}-14{ }^{\circ} \mathrm{C}$. Furthermore, the data reduction showed that all nights used for photometric observations were indeed photometric.

By the end of the observations more than $1500 \mathrm{CCD}$ frames were taken. The data reduction was performed by using the MIDAS image processing software, while in some cases, we also used DAOPHOT. It is evident that a bias offset has been subtracted and a flat-field correction has also been performed.

\section{Astrometric data reduction}

The night of April 26 was chosen for the astrometric part of this work, due to the good seeing value at Cerro La Silla of far below one arcsecond. Good seeing produces stable star traces of high quality, which enable an accurate determination of the right ascension direction. As it has been already mentioned, the angular separation $(\rho)$ as well as the position angle $(\theta)$ for every binary was calculated in the $V$ filter only on frames taken in this night.

In order to define the right ascension direction on the CCD chip, several trails of stars in M 16 open cluster were taken during this night using the $V$ filter. Obviously, all the CCD surface was used for taking these traces. In an earlier work, Sinachopoulos (1988) presented the technique of star traces for CCD cameras. Using several star trails per frame on six different frames taken one after the other, we determined the zero point of the position angle with an accuracy of $\sigma_{\theta}= \pm 0.005$ degrees.
In order to estimate the scale of the CCD we used several stars on images of M 16 taken in the same night of April 26 through the $V$ filter as well. A detailed astrometric work on this cluster has been published in the paper of Tucholke et al. (1986). According to it the typical internal errors of $\alpha$ and $\delta$ positions are both $00^{\prime \prime} 03$. The minimum accuracy (for $\delta=0$ ) in the position of one star, is

$$
\begin{aligned}
\sigma_{\mathrm{pos}} & =\sqrt{\left(\sigma_{\alpha} \cos \delta\right)^{2}+\left(\sigma_{\delta}\right)^{2}} \simeq \sqrt{\left(\sigma_{\alpha}\right)^{2}+\left(\sigma_{\delta}\right)^{2}} \\
& =\sigma_{\delta} \sqrt{2}
\end{aligned}
$$

Since the error in the angular separation of two stars of the M 16 cluster depends on the accuracy in the position of each one of them, the standard deviation will be

$$
\begin{aligned}
\sigma_{\mathrm{dd}} & =\sqrt{\left(\sigma_{\mathrm{pos} 1}\right)^{2}+\left(\sigma_{\mathrm{pos} 2}\right)^{2}}=\sqrt{2} \sigma_{\mathrm{pos} 1}=2 \sigma_{\delta} \\
& =0^{\prime \prime} 06 .
\end{aligned}
$$

According to the same paper, the mean error in the proper motion for the stars observed is 1 ." $4 / 1000 \mathrm{yrs}$ in both components. Since the difference of time between the epoch of the observations and the epoch to which the paper refers is $\Delta t=1994.4-1950=44.4$ years, the error in both components is

$\sigma_{\mu_{\delta}}=\sigma_{\mu_{\alpha \cos \delta}}=0^{\prime \prime} 0616$

and as we are interested in the angular separation of two stars, the error due to the proper motion uncertainty will be

$\sigma_{\mathrm{p} . \mathrm{m} .}=00^{\prime \prime} 1232$.

Moreover the error in the difference in the position of two stars does not depend on the error in the proper motion, the standard deviation in the angular separation will be

$\sigma_{\rho}=\sqrt{\left(\sigma_{\mathrm{dd}}\right)^{2}+\left(\sigma_{\mathrm{p} . \mathrm{m} .}\right)^{2}}=0^{\prime \prime} 137$.

We used several star pairs in M16 having angular separation around 600 pixels on the $\mathrm{CCD}$ chip, in order to determine the telescope scale with high accuracy. The error we derived for the scale factor in that way is

$\sigma_{\text {scale }}=\frac{\sigma_{\rho}}{600} \simeq 0.00022(" /$ pixel $)$.

By comparing the positions of the stars detected by DAOPHOT with the values presented in the paper mentioned above we calculated the scale factor of our optical system, which was $s=0.46249 \pm 0.00022^{\prime \prime} /$ pixel $(27 \mu /$ pixel). This agrees with the scale calculated for this telescope by van Dessel \& Sinachopoulos (1993).

For the error of the angular separation $\rho$ due to the atmospheric refraction we took $\sigma_{\mathrm{r}}(\rho)=0$. However, this is a rather poor assumption, since this error can be up to a few hundredths of an arcsecond for observations made 
at airmasses $X \approx 2$ if the $V$ filter is used and even higher for the $B$ and $U$ ones. Nevertheless, $X \approx 2$ was never the case of our astrometric observations, as they carried out near minimum possible airmasses (usually $X \leq 1.2$ ).

A discussion of the calculation of the final accuracy achieved in the angular separation was presented by Nakos et al. (1995) and a detailed one by Sinachopoulos \& Seggewiss (1990).

Although the method of star traces was used for the determination of the right ascencion direction, the good quality of the M 16 astrometry would enable us to use it for this purpose as well. A comparison of the two methods would be very interesting.

Table 1 contains the astrometric results. The first column is an enumeration of the visual binaries. The next four columns contain the coordinates of the primaries for epoch 2000. Next column contains the Durchmusterung designation of the primaries. The next four columns contain the angular separation $\left(\rho^{\prime \prime}\right)$, its accuracy $\left(\sigma_{\rho}\right)$ in arcsecs and the position angle $(\theta)$ with its accuracy $\left(\sigma_{\theta}\right)$ in degrees. The last column contains the epoch of the observations. It must be taken into account that the very small values of $\sigma_{\rho}$ errors are due to the assumption of $\sigma_{\mathrm{r}}(\rho)=0$. Realistic values of $\sigma_{\rho}$ are probably one order of magnitude higher.

\section{Photometric data reduction}

Photometric reduction consists of three parts: (a) the reduction in the instrumental photometric system (b) the data correction for the atmospheric extinction and (c) the transformation to the standard photometric system. For every new step of the reduction new errors are introduced and therefore the final accuracy of the results will decrease inevitably.

Two two-dimensional Moffat profiles were fitted simultaneously to the double star components on each CCD frame according to the classical least square technique (Cuypers 1994).

The components' magnitude difference resulted from this on chip instrumental photometry had always an accuracy of a few millimagnitudes. On the other hand, all nights proved to be of good photometric quality as well. These two factors enabled us to get nightly instrumental magnitudes of the components of the observed double stars with an accuracy of 0.004 mags or better.

The atmospheric extinction correction was derived according to the classical formulas

$$
\begin{aligned}
v_{0} & =v-\left(k_{v}^{\prime}+k_{v}^{\prime \prime}(b-v)\right) \cdot X \\
(b-v)_{0} & =(b-v)-\left(k_{b v}^{\prime}+k_{b v}^{\prime \prime}(b-v)\right) \cdot X \\
(u-b)_{0} & =(u-b)-\left(k_{u b}^{\prime}+k_{u b}^{\prime \prime}(u-b)\right) \cdot X
\end{aligned}
$$

where the " 0 " index refers to the mangitude and the color indices outside the atmosphere. We should remind the $v$ mangitude and the $b-v$ and $u-b$ color indices refer to the instrumental values.

For the determination of the first order extinction coefficients we used the A0 - type E408 standard star from the E regions (Graham 1982), which was observed each night, many times per night at different airmasses smaller than or equal to two. The first order extinction coefficients that we obtained are the following:

$$
\begin{array}{ll}
25 / 4 / 1994 & k_{v}^{\prime}=0.1225 \pm 0.0017 \\
25 / 4 / 1994 & k_{b}^{\prime}=0.2305 \pm 0.0017 \\
25 / 4 / 1994 & k_{u}^{\prime}=0.4592 \pm 0.0014 \\
26 / 4 / 1994 & k_{v}^{\prime}=0.1172 \pm 0.0020 \\
27 / 4 / 1994 & k_{b}^{\prime}=0.2339 \pm 0.0023 \\
28 / 4 / 1994 & k_{u}^{\prime}=0.4526 \pm 0.0016 \\
30 / 4 / 1994 & k_{v}^{\prime}=0.1261 \pm 0.0022 \\
01 / 5 / 1994 & k_{b}^{\prime}=0.3431 \pm 0.0086
\end{array}
$$

This list shows as well which filter was used each night for carrying out the observations.

Second-order extinction coefficients of the $U B V$ system are fairly constant and can be calculated only twice per year for each observatory. For this reason, we used the values given by Gocherman et al. (1993) as updated by Grothues (1995).

For the transformation in the standard system, we used the average and the standard deviation values of the $V$ extinction corrected magnitude and of the $(b-v)$ extinction corrected colour. These were calculated from the two $V$ and the two $(b-v)$ extinction corrected values, which were obtained in the corresponding two pairs of two different nights of observations with the same filter. As we observed only one night in $U$ filter, we calculated the error of the $(u-b)$ extinction corrected colour according to the corresponding error equation.

The three standard deviation values of these extinction corrected magnitudes and colours, were around 0.01 mags.

The $V$ magnitude and the $B-V$ and $U-B$ color indices, which refer to the standard system, can be obtained from the extinction corrected values $v,(b-v)$ and $(u-b)$ according to the following transformations:

$$
\begin{aligned}
V & =v+\epsilon \cdot(B-V) \\
(B-V) & =\mu \cdot(b-v) \\
(U-B) & =\psi \cdot(u-b) .
\end{aligned}
$$

For the estimation of $\epsilon, \mu$ and $\psi$ we used improved photometric values of 18 photometric standard stars from the E regions (Grenon 1991) observed in the night of the 
$25^{\text {th }}$ to $26^{\text {th }}$ of April. The values obtained for the transformation coefficients are the following:

$$
\begin{aligned}
& \epsilon=0.033 \pm 0.003 \\
& \mu=1.047 \pm 0.008 \\
& \psi=0.949 \pm 0.008 .
\end{aligned}
$$

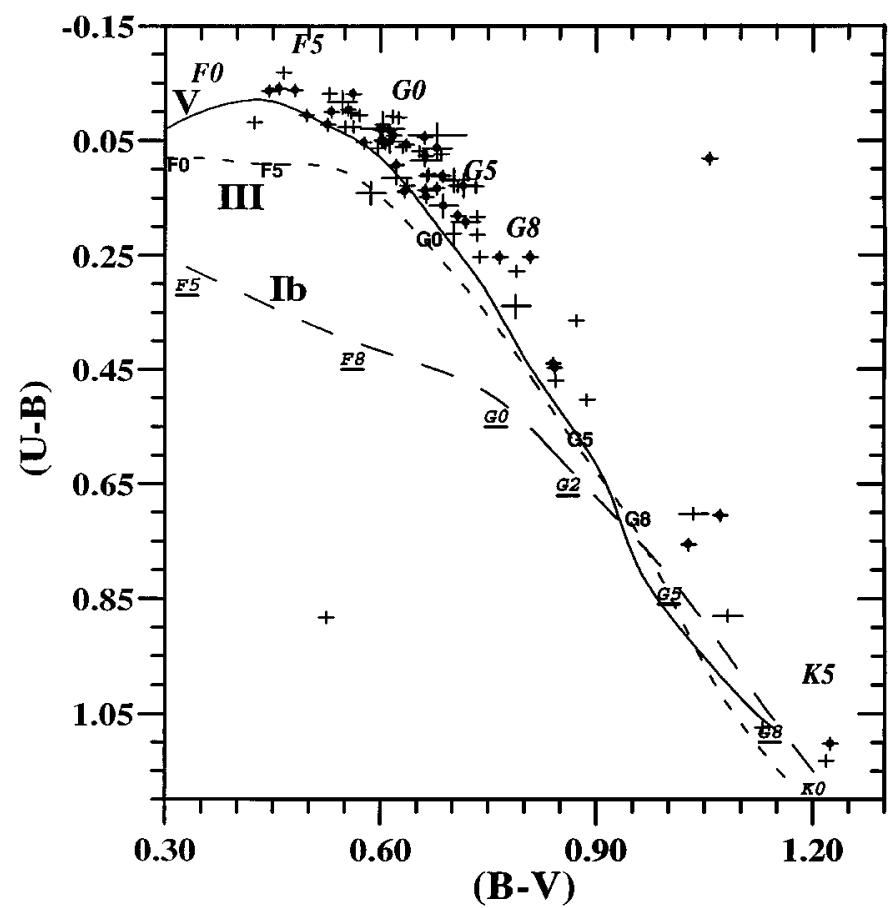

Fig. 1. The two colour diagram

Table 2 contains the photometric results of the reduction that was performed on 40 visual binaries. Two lines per binary are given. The first corresponds to $V$ magnitude and colours of the primary while the second one of the secondary. As in Table 1, the first column is an enumeration of the binaries, while the second column contains the Durchmusterung designation of the primaries. The next three columns contain the $V$ magnitude and its error $\sigma_{V}$ and the $(B-V)$ and $(U-B)$ color indices with their errors $\sigma_{B-V}$ and $\sigma_{U-B}$ respectively.

The error estimations in Table 2 resulted from the standard deviation values of the extinction corrected magnitudes and colours and the uncertainties of the transformation coefficients into the standard $U B V$ system.

\section{Discussion}

In Fig. 1 we present the two color diagram of the observed stars. The encircled-cross sign refers to the primaries, while the simple-cross refers to the secondaries.
Table 3. Photometric differences and angular separation of the components

\begin{tabular}{|r|ll|l|c|r|r|}
\hline No & ADS & or DM & $\rho\left[^{\prime \prime}\right]$ & $\Delta V$ & $\Delta(B-V)$ & $\Delta(U-B)$ \\
\hline 1 & -48 & 4325 & 5.345 & 0.593 & 0.068 & 0.236 \\
2 & -03 & 2577 & 5.509 & 1.062 & 0.170 & 0.210 \\
3 & -24 & 8146 & 2.168 & 1.010 & 0.058 & 0.057 \\
4 & -07 & 2793 & 4.286 & 0.445 & 0.090 & 0.044 \\
5 & -57 & 2434 & 2.559 & 0.038 & 0.102 & 0.318 \\
6 & -01 & 2347 & 2.731 & 0.059 & -0.004 & 0.033 \\
7 & -58 & 2243 & 5.146 & 0.016 & 0.003 & 0.031 \\
8 & -40 & 6603 & 2.009 & 0.069 & 0.114 & 0.039 \\
9 & -40 & 6622 & 2.296 & 0.474 & 0.064 & 0.019 \\
10 & -74 & 804 & 1.545 & 0.244 & -0.063 & -0.153 \\
11 & -46 & 7356 & 3.757 & 1.327 & 0.071 & 0.087 \\
12 & -13 & 3466 & 3.301 & 0.203 & 0.029 & 0.030 \\
13 & -23 & 10415 & 6.574 & 1.845 & -0.006 & 0.029 \\
14 & -34 & 7899 & 1.627 & 0.041 & -0.008 & -0.013 \\
15 & -62 & 2853 & 2.319 & 0.278 & 0.032 & -0.025 \\
16 & -47 & 8078 & 3.779 & 0.056 & 0.023 & 0.003 \\
17 & -57 & 6065 & 3.153 & 0.040 & -0.033 & -0.001 \\
18 & -29 & 10446 & 2.230 & 0.121 & 0.043 & -0.018 \\
19 & -26 & 9458 & 2.733 & 0.269 & 0.023 & 0.087 \\
20 & -27 & 9212 & 2.902 & 0.344 & 0.007 & -0.028 \\
21 & -79 & 740 & 5.456 & 0.106 & -0.092 & 0.078 \\
22 & -31 & 10930 & 4.415 & 0.302 & 0.261 & 0.334 \\
23 & +04 & 2864 & 2.737 & 0.042 & 0.040 & -0.045 \\
24 & -16 & 3942 & 4.615 & 0.021 & 0.357 & 0.569 \\
25 & -14 & 4070 & 3.487 & 0.075 & 0.046 & 0.052 \\
26 & -25 & 10904 & 4.189 & 0.139 & 0.023 & 0.030 \\
27 & -31 & 12112 & 2.031 & 0.152 & 0.026 & 0.034 \\
28 & -38 & 10617 & 2.186 & 0.037 & 0.000 & -0.007 \\
29 & -04 & 3976 & 3.704 & 0.072 & 0.045 & 0.056 \\
30 & -28 & 12218 & 2.506 & 0.185 & 0.070 & -0.006 \\
31 & -65 & 3193 & 1.915 & 0.470 & -0.533 & 0.801 \\
32 & -17 & 4630 & 3.404 & 0.195 & 0.009 & 0.064 \\
33 & -46 & 11189 & 2.264 & 0.332 & 0.064 & 0.059 \\
34 & -46 & 11604 & 2.918 & 0.138 & 0.003 & -0.010 \\
35 & -13 & 4639 & 2.352 & 0.294 & 0.048 & 0.071 \\
36 & -52 & 10777 & 4.626 & 0.237 & 0.043 & 0.043 \\
37 & -04 & 4202 & 1.905 & 0.011 & -0.013 & -0.010 \\
38 & -57 & 8803 & 2.831 & 0.561 & 0.275 & 0.628 \\
39 & -32 & 15843 & 1.867 & 0.395 & 0.074 & 0.104 \\
40 & -32 & 13472 & & 2.349 & -0.648 & -0.687 \\
\hline
\end{tabular}

The lengths of the cross lines correspond to the accuracy of the results. The curves which are also present refer to the Keenan-Morgan spectral classification for dwarf, giant and supergiant $\mathrm{F}$ and $\mathrm{G}$ - type stars. As it can be easily seen, both components of most systems are G-type main sequence stars.

In Table 3 we present the angular separation as well as the magnitude and colour differences between the two components of each system. In eight cases (Nos. 6, 7, 13, $14,20,28,32$ and 34$)$, the $\Delta(B-V)$ color index is of the order of some millimagnitudes. Since the accuracy of the photometric results is of the order of a hundredth of 
a magnitude (Table 2) we can practically assume that the components of these eight systems have the same effective temperature. In almost all cases (system No. 13 is excluded) $\Delta V$ is much less than one magnitude, while the angular separation of the components is less than six arcseconds. By taking under consideration the Aitken limit as well, we conclude that these seven systems are physical pairs and not just optical pairs.

In the case of system " $-48^{\circ} 4325$ " both components must be of B- type. Due to the interstellar reddening, however, their position in the two color diagram was shifted downwards. This is the reason for which two points with very negative $(U-B)$ indices are present in the two color diagram.

From the results obtained from the 40 binaries presented here, it is not possible to derive a good estimation of the width of the main sequence, for the G-type stars. This will be the main goal of our forthcoming activities.
Acknowledgements. This research was mainly carried out in the framework of the project "Service Centres and Research Networks", initiated and financed by the Belgian Federal Scientific Services (DWTC/SSTC).

\section{References}

Cyupers J., 1993 (private communication)

Gocherman J., et al., 1993, A\&AS 99, 591-614

Graham J.A., 1982, PASP 424, 244-263

Grenon M., 1991 (private communication)

Grothues H.-G. (personal communication)

Nakos Th., Sinachopoulos D., van Dessel E., 1995, A\&AS 112, 453-456

Rakos K.D., et al., 1982, A\&AS 47, 221-235

Sinachopoulos D., 1988, A\&AS 76, 189

Sinachopoulos D., Seggewiss W., 1990, A\&AS 83, 245

Tucholke H.-J., Geffert M., Thé P.S., 1986, A\&AS 66, 311-322

van Dessel E., Sinachopoulos D., 1993, A\&AS 100, 517-520 